\title{
Efficacy of Emotion Regulation Skills and Meta-cognitive Beliefs in Reducing Social Phobia Among Students- A Casual-Comparative Study
}

\author{
Esmaiel Soleymani ${ }^{1}$, Azime Ekhtiyari ${ }^{2}$, Mojtaba Habibi ${ }^{3}$, Mani B. Monajemi ${ }^{4,}$, \\ ${ }^{1}$ Department of Psychology, Urmia University, Urmia, Iran \\ ${ }^{2}$ School of Psychology, Ardebil Azad University, Ardebil, Iran \\ ${ }^{3}$ Department of Health Psychology, Tehran Institute of Psychiatry- School of Behavioral Sciences and Mental Health, Iran University of \\ Medical Sciences, Tehran, Iran \\ ${ }^{4}$ Department of Psychology and Mental Health Sciences, University of Tehran, Tehran, Iran
}

\section{Email address:}

mani.b.monajemi@warwickgrad.net (M. B. Monajemi), mani.b.monajemi@ut.ac.ir (M. B. Monajemi)

${ }^{*}$ Corresponding author

\section{To cite this article:}

Esmaiel Soleymani, Azime Ekhtiyari, Mojtaba Habibi, Mani B. Monajemi. Efficacy of Emotion Regulation Skills and Meta-cognitive Beliefs in Reducing Social Phobia Among Students- A Casual-Comparative Study. International Journal of Psychological and Brain Sciences. Vol. 2, No. 1, 2017, pp. 10-17. doi: 10.11648/j.ijpbs.20170201.12

Received: July 24, 2016; Accepted: September 12, 2016; Published: February 27, 2017

\begin{abstract}
The purpose of this study was to compare the efficacy of emotion regulation skills and meta-cognitive beliefs in reducing social phobia among students. The study method was experimental type. The statistical society of this study was consisted of 60 high school girls in Tehran, who were selected randomly as case group and were divided evenly into three groups (each group 20 individuals). Emotion regulation and meta-cognition training were considered for both groups for 8 sessions and one group was selected as control. Data were collected using the Social Phobia Inventory (SPIN). Obtained information was analyzed via MANCOVA and the SPSS software. The results were demonstrative of reduced symptoms in social phobia and panic symptoms, avoidance and physiological symptoms in the case group $(p<0.001)$ after receiving emotion regulation skills and meta-cognitive training. Tukey test showed effectiveness of two methods (Emotion regulation and meta-cognition). It appears plausible that emotion regulation and meta-cognitive interventions can be used to improve the symptoms of social phobia.
\end{abstract}

Keywords: Emotion Regulation, Meta-cognitive Beliefs, Social Phobia

\section{Introduction}

Social phobia includes persistent fear of negative evaluation by others, which is considered one of the subtypes of anxiety disorders. People with social phobia are extremely afraid of humiliation and shame in certain social situations, such as speaking in public. This fear may include concern from incidence of anxiety symptoms such as blushing, trembling and sweating (the psychology of America, 2000). Disorder of social phobia or anxiety is classified as one of the anxiety disorders as and the third psychiatric disorder in terms prevalence between general populations (Hufman \& Barlow, 2002). This disorder is recognized based on the fear from social $t$ situations. The prevalence rate of social phobia among adolescents is from 5 to 13 percent. All cognitive theories have referred on the role of an important factor in creating and maintaining social anxiety disorder and it is the biased processing of information (Rapee \& Spence, 2004).

Meta-cognitive ability is of the cognitive processes, which seems to be flawed among people with social phobia. Metacognition-psychology is a new concept that its history goes back to the 1970s. Flavell was first man who coined the term meta-cognition in 1979. It seems that meta-cognition includes both cognitive processes, experiences and cognitive regulation. Meta-cognitive knowledge refers to the acquisition of knowledge about the cognitive processes and knowledge about how to use cognitive control processes. Metacognition is a broad term, encompassing both knowledge and regulation of cognitive activity (Moses \& 
Baird $\left.^{1}, 2002\right)$. In a sense, it can be considered as a generic aspect of cognition, which is involved in all cognitive activities. Some specific aspects Meta-cognition associated with psychological disorders (Wells \& Matthews ${ }^{2}$, 1994; Wells, 2003). Meta-cognition is a multifaceted concept, which involves knowledge (beliefs) processes and strategies that controls recognition, assessment and monitoring (Moses \& Baird, 2002). Lobban, Haddock, Einderman and Wells (2002) based on a research noted that meta-cognitive beliefs in patients with anxiety disorders especially in generalized anxiety, panic, obsessive-compulsive and post-traumatic stress have a further disorders. Meta-cognition forms what we consider and enter the domain of our consciousness, evaluations and the effect of different strategies that we use to adjust our thoughts and feelings (Wells, 2009).

The study of meta-cognitive processes involved in psychiatric disorders is basically relevant with the theory of self-regulatory enforcement function ${ }^{3}$ (S-REF) Wells and Matthews (1994). The central concept in the S-REF is that maintains emotional disorder, consequently, a wide range of it is mentioned the activation of schemas and comprises attention processes, the situations of self-regulatory purpose, meta-cognition and processing styles (Wells, 2003). Metacognitive processes have two independent aspects but related to each other: Meta-cognitive knowledge and meta-cognitive experience (Kadivar, 2004), meta-cognitive strategies are used to monitor cognitive strategies and lead them. The results of several studies have shown meta-cognition intervention improves anxiety symptoms in patients with social phobia and improvement of phobic symptoms on the exam anxiety (Bahadori et al., 2011; Vaziri \& Mousavi, 2008; Momeni et al., 2013).

When people with high anxiety sensitivity confronted with greater challenges in regulating their emotional functions are less able to recognize and accept their emotions and they are more willing to show negative emotions in ambiguous emotional situation (Kashdan et al., 2006). Recent evidence regarding anxiety disorders indicates that difficulties in emotion regulation may be an important factor in these disorders (Rodebaugh \& Heimberg, 2008). Barlow (2002) is discussed control perceptions over anxiety as an important determinant factor for etiology and persistence of anxiety disorders. People with social anxiety disorder experience the unexpected and continuous experience of the thrills, these subsequent emotional warnings in vulnerable people causes to know their physical and emotional reactions uncontrollable. Therefore, they believe to avoid social situations, because they predict the lack of internal control over their emotional response when facing with social anxiety situations (Hoffman, 2005). It seems that the abilities to identify and describe emotional experiences are aspects of emotion regulation that associated with social anxiety. Defects in these areas in terms of structural problems are

\footnotetext{
1 - Moses \& Baird

2 - Wells \& Matthews

3 -Self- Regulatory Exective Function
}

explained in the cognitive emotion regulation. There are various definitions of emotion regulation, but all of them have defined it as the manner in which individuals control their experience and expression of emotion under distress conditions using strategies such as supervision, suppression and cognitive reappraisal (Gross, 2002). Studies show emotion regulation is from psychological characteristics that often associated with anxiety sensitivity (McDermott et al, 2009; Tull et al, 2009). Jacobs et al (2008) showed that high emotional understanding; appropriate uses from emotions and positive emotional experience have a significant negative correlation with severity of social anxiety in people with the disease, so that there is an inverse relationship between levels of social anxiety and self-referral emotional processing levels. Tal et al (2009) found that individuals with panic and generalized anxiety disorder have numerous problems in regulating their emotions. Research results have shown interventions of emotional regulation are created to reduce anxiety, mental health, self efficacy and emotion (Mashhadi et al., 2013; Momeni et al., 2013; Narimani et al., 2011; Gross \& John, 2003). Along conducted researches, the research seeks to answer the question that do the effects of emotion regulation strategies and meta-cognitive beliefs in reducing the symptoms of social phobia of the students is different?

\section{Method}

The research method in this study given the goals and assumptions is the type of experimental. Multi-group pretest and posttest project with control group was used. In this study, researchers compared the effects of emotion regulation and cognitive strategies on reducing social phobia of students. Teaching emotion regulation and meta-cognitive strategies in this research is considered as the independent variable and social phobia as the dependent variable.

\subsection{Society, Sample and Sampling Methods}

The statistical society of the research included girls in high school in Bilehsuar in -2014-2015 that based on the information obtained from Education District in Bilehsuar, 800 female students are studying in this area. The random sampling method was used in this study. Thus, the implementations of social phobia scale SPIN on 600 students from schools, 85 people were identified as students with social anxiety disorder on the basis of cut-off point higher than score 40 and then, 60 of these were selected randomly and were replaced in one of three research groups (Department emotion regulation, education, meta-cognitive strategies, and control). Thus, 20 patients in each experimental group and 20 patients were placed in the control group. As regards the number of participants in pilot projects are at least 15 people, in order to increase external validity and generalization capability with more confidence, 20 patients and in total, 60 subjects were selected (Delaware, 2006). 


\subsection{The Following Instruments Were Used to Collect Data}

Social Phobia Inventory (SPIN) first was prepared by Connor and colleagues to assess social anxiety or social phobia. Clinical implications suggest that provide information in three areas of clinical signs of fear, avoidance and physiological signs and have practical advantages of being short, simplicity and ease of scoring. This tool having stable psychometric properties can be used as a valid tool for assessing the severity of symptoms of social phobia. Also can be used as a screening tool and to test the response to treatment of social anxiety disorder, finally, this scale can be distinguished treatments with different performance from each other (Connor et al., 2000). This questionnaire has high reliability and validity. Its reliability with test retest in groups with the diagnosis social anxiety disorder was 78 to 89 percent and its coefficient of internal consistency (coefficient alpha) in a normal is reported 94 percent. Also it is reported for Sub-scale for fear $89 \%$, for avoiding $91 \%$ and physiological discomfort was $80 \%$. Construct validity compared with the test results in two groups of subjects with the diagnosis panic disorder and normal subjects with no psychiatric diagnosis showed a significant difference which this suggests a high construct validity. SPIN is a 17-item selfreports scale that has three fear sub-scales (6 materials), avoidance (7 materials) and psychological distress (4 materials) where each material is graded based on a fivepoint Likert scale $(0=$ in any way, $1=$ low, $2=$ somewhat, 3 = high, $4=$ very much). Scores for each of the subscales are obtained through sum of the scores on materials related to its subscale. Based on the results, to interpret the scores, people with social phobia are distinguished from non-infected based on cut-off point of 40 with $80 \%$ recognition accuracy performance and cut-off point of 50 with $89 \%$ recognition accuracy performance (Connor et al., 2000; translated FathiAshtiani et al., 2013). Descriptive statistics of mean and standard deviation, etc to analyze the data from this study and also to evaluate the research hypotheses, MANCOVA (to control pre-test) and Tukey test was used to compare the two groups.

\subsection{Implementation Method}

Data collection in this research was fieldwork. In this study, to gather the needed information, after obtaining the necessary permits go to schools and SPIN was placed at the disposal of 600 students. After completion, students who have achieved score of 40 or higher on scale social anxiety were considered as people with social phobia. Then from 85 students who have been diagnosed with social phobia, 60 students were selected simple randomly and were placed in two groups of 20 people experiment and a group of 20 control. Then the objectives and research project explained for the people and after satisfying these individuals to participate in the questionnaire, SPIN social phobia was placed at the disposal of these people and were considered as a pre-test after completing, and then the first group spent eight one and half hour session intervention of emotion regulation and the second group eight one and half hour session intervention of meta-cognitive strategies. The control group spent their daily routine. After completing the sessions again, questionnaire of social phobia was placed at the disposal of the people to answer and were considered as a post-test. And the results were analyzed using SPSS software.

\subsection{Intervention Method}

\subsubsection{Intervention Methods of Emotion Regulation}

First session: Preparation, primary communication, referrals, explain the purpose of the study, the concept of the problem, satisfy the people to participate in research projects, questionnaire of primary interviews for pre-test, determine the time and place (two sessions per week) holding next intervention meetings.

Second session: In the second session were comments on the excitement and with a brief overview of contents the previous session, comments on the emotion regulation were discussed. And also explanations were given about describes benefits of emotion regulation: emotions regulation and control have at least two important benefits: 1- It is very useful for mental and physical health. 2- It is an important social skill.

Third session: After a brief review of prior session, the excitement dimensions were described: Mental dimension makes to feel such as angry or happy a specific way. Biological dimension: It means the answers to mobilize the energy that body provides to adapt to any situation person face with it. Target dimension: Namely the creation of motivational desire to do anything, for example, anger creates motivation to fight or protest with injustice in our, otherwise we do not. Social dimension: We send signs such as face recognizable, gesture and verbal when we get excited, that will inform others about the quality and intensity of our excitement for example, eyebrows and tone of voice.

Fourth session: During the session after a brief review of previous session, fundamental skills of disciplining emotional were described: Your excitements: what they are? How do they operate? Survival ("fight or flee"), remembering people and situations, deal with the problems of everyday life, relationships with others, avoidance of pain, pleasureseeking.

Fifth session: In this session researcher pays to describe these skills: What are the skills that bring discipline to excitement? Know your emotions, overcoming the barriers to healthy emotions, reduce your physical and cognitive vulnerability; increase your positive emotions, without judgment to your emotions, show mindfulness, face with your emotions, act conversely your emotional desire and solve the problems.

Sixth session: In this session after a brief review of previous session, the discussions were focused about overcoming barriers to healthy emotions. In this session, the subjects were told: Now that you start more accurate identity of your excitements, we hope that you will discover how the effects of emotions on your thoughts and behaviors. In the following session explanation was given about emotions and 
behavior of individuals and the damage to self.

Seventh session: In this session to provide explanations and emotion regulation skills was discussed: Skills: observing without judging ourselves, cognitive vulnerability reduction, the use of coping thoughts and also training will be given about creating a balance between thoughts and feelings.

Eighth session: In this session, skills of observing without judging ourselves, cognitive vulnerability reduction, the use of coping thoughts and creating a balance between thoughts and feelings to be trained and the subjects were asked to use their learning during exercise sessions in their daily life. And at the end of the session - a half hour was spent to run the posttest. (Content validity of the curriculum by has been approved by experts in the field of psychology (dissertation supervisor).

\subsubsection{Intervention Method Meta-cognitive Strategies}

Meta-cognitive intervention in this study was used based on meta-cognition treatment for anxiety disorders.

First session: Introduction, evaluation of symptoms and introduction of social anxiety disorder, separation of normal social anxiety and troublesome and problematic for patients, determine the need for treatment.

Second session: Presentation the meta-cognitive treatment logic for social anxiety disorder, investigation of anxiety symptoms in social panic disorder, investigation of effective possible causes in the etiology of social anxiety disorder, investigation of different treatments for social anxiety disorder and providing explanations about the logic of metacognitive treatment for social anxiety disorder.

Third session: Evaluation and identification of positive and negative meta-cognitive beliefs in patients and analysis of advantages and disadvantages of these beliefs.

Fourth session: Identification of meta-cognitive control strategies in patients, analyze the advantages and disadvantages of these strategies and replace more efficient meta-cognitive strategies.

Fifth session: Application of way away from the mind, knowledge in dealing with dysfunctional meta-cognitive strategies and thoughts (Patients learn to deal with thoughts like a cloud in their mind if the processing is not required.

Sixth session: Familiarize patients with two strategies of anxiety and rumination as dysfunctional coping strategies, analysis of profit and loss anxiety and rumination, analysis of suppressing thoughts of as an inefficient process (White Tiger) and familiarization with the consequences of infection with symptoms training anxiety retard way.

Seventh session: Familiarize patients with significant cognitive symptoms in continuity of mental disorders, Presentation logic of ATT to patients and teaching the styles them.

Eighth session: Training focus way attention on the situation as an effective meta-cognitive strategy and preparing members for closing, identify barriers to the use of methods, finding the cause and eliminate it and finally concluded. In each session, homework was given in addition to the previous session review and practices and the subjects were asked touse their learning during sessions in their daily life. And at the end of the session - a half hour was spent to run the posttest. (Content validity of the curriculum by has been approved by experts in the field of psychology (dissertation supervisor).

\subsection{Findings}

Table 1. Mean and standard deviation scores of social phobia, fear, avoidance, physiological symptoms in three groups

\begin{tabular}{|c|c|c|c|c|c|c|c|}
\hline \multirow{2}{*}{ Variables } & \multirow{2}{*}{$\begin{array}{l}\text { Type of } \\
\text { Test }\end{array}$} & \multicolumn{4}{|l|}{ Experiment } & \multicolumn{2}{|c|}{ Control } \\
\hline & & Meta-Cognition & & Emoti & Ilation & Mean & Standard Deviation \\
\hline \multirow{2}{*}{ Social Phobia } & Pretest & 58.05 & 4.23 & 58.15 & 4.06 & 58.5 & 2.41 \\
\hline & Posttest & 36.05 & 4.22 & 38.8 & 4.42 & 51.8 & 2.64 \\
\hline \multirow{2}{*}{ Fear } & Pretest & 20.95 & 1.50 & 20.8 & 1.73 & 22.1 & 1.33 \\
\hline & Posttest & 13 & 2.42 & 13.8 & 1.70 & 18.65 & 1.56 \\
\hline \multirow{2}{*}{ Avoidance } & Pretest & 23.40 & 2.25 & 23.85 & 1.72 & 23.55 & 1.23 \\
\hline & Posttest & 14.45 & 2.23 & 16.3 & 2.27 & 21.55 & 1.50 \\
\hline Psychological Symptoms & Posttest & 8.6 & 1.39 & 8.7 & 1.34 & 12.7 & 0.80 \\
\hline
\end{tabular}

Results Table 1 shows in the social phobia scores, the control group with the mean (58.5) achieved the highest and emotion regulation group with the score (58.05) had the lowest average in the pre-test. And in the posttest control group with the score (51.8) the highest and emotion regulation group with the score (36.05) had the lowest average. In the fear scors control group with the score (22.1) the highest and emotion regulation group with the score (20.95) had the lowest average in the pretest. And in the posttest control group with the score (18.65) the highest and meta-cognition group with the score (20.08) had the lowest average. In avoidance scores, the meta-cognition group with the score (23.85) the highest average and emotion regulation group with the score (23.40) had the lowest average in pretest. And in the posttest control group with the score $(21.55)$ the highest and emotion regulation group with the score (14.45) had the lowest average. Physiological symptoms scores, control group with the score (14.95) the highest and meta-cognition group with the score (13.50) had the lowest average in the pretest. And in the posttest control group with the score (12.7) the highest and emotion regulation group with the score (8.6) had the lowest average. 
Table 2. Results of Levine tests to check the homogeneity of scores variance between social phobia, fear, avoidance and physiological symptoms.

\begin{tabular}{lllll}
\hline Groups & F & df $^{\mathbf{1}}$ & df $^{\mathbf{2}}$ & Significance \\
\hline Social Phobia & 1.61 & 2 & 57 & 0.20 \\
Fear & 0.97 & 2 & 57 & 0.38 \\
Avoidance & 1.46 & 2 & 57 & 0.23 \\
Psychological symptoms & 0.26 & 2 & 57 & 0.76 \\
\hline
\end{tabular}

The results of Table 2 show that the Levine test is not significant. Based on these results, homogeneity defaults of variance in these variables were approved in studied groups. This test was not significant for any of the variables. As a result, the use of parametric tests, multivariate analysis of variance is allowed.

Table 3. Results of multivariate variance analysis tests on the scores means of social phobia, fear, avoidance and physiological symptoms.

\begin{tabular}{llllllll}
\hline \multirow{4}{*}{ Group } & Amount & F & df $^{1}$ & df $^{2}$ & Significance & Chi Eta \\
\cline { 2 - 7 } & Pillai effect & 0.81 & 12.74 & 6 & 112 & 0.000 & 0.40 \\
& Wilks' Lambda & 0.20 & 21.98 & 6 & 110 & 0.000 & 0.54 \\
& Hotteling effect & 3.74 & 33.73 & 6 & 108 & 0.000 & 0.65 \\
& The root of the error & 3.72 & 69.51 & 6 & 56 & 0.000 & 0.78 \\
\hline
\end{tabular}

Table 3 shows the results of significant test of MANCOVA based on social phobia, fear, avoidance and physiological symptoms in both experimental and control groups. Results show that there is a significant difference between students with symptoms of social phobia in terms the variables in two experimental groups and one control group. Eta-square (which actually is the square of the correlation coefficient between the dependent variables and group membership) shows that the difference between three groups considering to the dependent variable is significant in total. And this difference is 0.54 percent according to Wilks lambda test. Namely $54 \%$ of the variance has been related to the difference between three groups due to independent variables influence hence strategies of emotion regulation.

Table 4. The results of covariance analysis to determine the effectiveness of the intervention emotion regulation on the scores of social phobia, fear, avoidance and physiological symptoms.

\begin{tabular}{lllllll}
\hline Statistical indices & SS & df & MS & F & Signification & Chi Eta \\
\hline Social Phobia & 2796.33 & 2 & 1398.16 & 95.23 & 0.000 & 0.77 \\
Fear & 305.04 & 2 & 152.52 & 40.57 & 0.000 & 0.59 \\
Avoidance & 542.33 & 2 & 271.16 & 64.54 & 0.000 & 0.69 \\
Psychological symptoms & 108.51 & 2 & 54.25 & 39.13 & 0.000 & 0.58 \\
\hline
\end{tabular}

As it can be seen in Table 4, the results of covariance analysis shows scores of social phobia $(\mathrm{F}=95.23), \mathrm{fear}(\mathrm{F}=40.57)$, avoidance $(\mathrm{F}=64.54)$ and physiological symptoms $(\mathrm{F}=39.13)$ is significantly higher in two experimental group than the control group $(\mathrm{P}<0.001)$. In other words, emotion regulation and meta-cognition intervention has reduced the symptoms of social phobia in the students.

Table 5. Results of Tukey test to determine differences between three groups in the scores of social phobia, fear, avoidance and physiological symptoms.

\begin{tabular}{|c|c|c|c|c|}
\hline Variables & Groups & Mean difference & Standard deviation & Significance \\
\hline \multirow{6}{*}{ Social Phobia } & Meta-Cognition & -2.65 & $1 / 59$ & 0.23 \\
\hline & Control & -19.55 & $1 / 59$ & 0.000 \\
\hline & Emotion Regulation & 2.65 & $1 / 59$ & 0.23 \\
\hline & Control & -16.9 & $1 / 59$ & 0.000 \\
\hline & Emotion Regulation & 19.55 & $1 / 59$ & 0.000 \\
\hline & Meta Cognition & 16.9 & 1.59 & 0.000 \\
\hline \multirow{6}{*}{ Fear } & Meta-Cognition & 0.95 & 0.73 & 0.40 \\
\hline & Control & -5.75 & 0.73 & 0.000 \\
\hline & Emotion Regulation & 6.7 & 0.73 & 0.000 \\
\hline & Control & 5.75 & 0.73 & 0.000 \\
\hline & Emotion Regulation & -0.30 & 0.40 & 0.74 \\
\hline & Meta Cognition & -4.85 & 0.40 & 0.000 \\
\hline \multirow{5}{*}{ Avoidance } & Meta-Cognition & 0.30 & 0.40 & 0.74 \\
\hline & Control & -4.55 & 0.40 & 0.000 \\
\hline & Emotion Regulation & 4.85 & 0.40 & 0.000 \\
\hline & Emotion Regulation & -1.40 & 0.85 & 0.23 \\
\hline & Meta Cognition & -8 & 0.85 & 0.000 \\
\hline \multirow{6}{*}{ Psychological Symptoms } & Meta-Cognition & 1.4 & 0.85 & 0.23 \\
\hline & Control & 6.60 & 0.85 & 0.000 \\
\hline & Emotion Regulation & 8 & 0.85 & 0.000 \\
\hline & Control & 6.6 & 0.85 & 0.000 \\
\hline & Emotion Regulation & 0.95 & 0.73 & 0.40 \\
\hline & Meta Cognition & -5.75 & 0.73 & 0.000 \\
\hline
\end{tabular}


As it can be seen in Table 5 the results of Tukey test shows that there is a significant difference between the control group and two meta-cognition training groups and emotion regulation $(\mathrm{P}<0.000)$. Also results show there was no significant results $(\mathrm{P}>0.000)$ between meta-cognition and emotion regulation groups. In other words, training emotion regulation and meta-cognition had no significant difference in the reduction of social phobic children.

\section{Discussion and Conclusion}

The purpose of this study was comparison of the effectiveness of emotion regulation strategies and metacognitive beliefs in reducing social phobic of the students. The first hypothesis of this study was: Emotion regulation strategies affect the reduction of student's social phobia. ANOVA was used to test the hypothesis. The test results indicated that the first hypothesis is confirmed $(\mathrm{P}<0.001)$, in other words, the results showed emotion regulation has significantly reduced the symptoms of social phobia and physiological symptoms, fear and avoidance of social phobia in the study group. The results of studies on emotion regulation shows emotion regulation is of psychological characteristics that often is associated with anxiety sensitivity (McDermott et al, 2009). Difficulties in emotion regulation may be an important factor in anxiety disorders (Rodebaugh $\&$ Heimberg, 2008). People with social disorder suffer from intense fear of social evaluation, increase emotional responses to social cues and face the basic problems in emotion regulation especially in social situations (Philip et al., 2014). The high emotional understanding; appropriate uses from emotions and positive emotional experience have significant negative correlation with severity of social anxiety in people with the social anxiety disorder, so that there is an inverse relationship between levels of social anxiety and self-referral emotional processing levels (Jacobs et al, 2008). Mashhadi et al (2013) in their research have shown re-assessment as one of the component of emotional regulation as significant negative and anxiety sensitivity as positive were able to predict social anxiety among students. Also individuals with panic and generalized anxiety disorder have numerous problems in regulating their emotions (Tal et al, 2009). When people with high anxiety sensitivity confronted with additional challenges in regulating their emotional functions are less able to recognize and accept their emotions and they are more willing to show negative emotions in ambiguous emotional situation (Kashdan et al, 2006). Barlow (2002) is discussed control perceptions over anxiety as an important determinant factor for etiology and persistence of anxiety disorders. People with social anxiety disorder experience the unexpected and continuous experience of the thrills, these subsequent emotional warnings in vulnerable people causes to know their physical and emotional reactions uncontrollable. Therefore, they believe to avoid social situations, because they predict the lack of internal control over their emotional response when facing with social anxiety situations (Hoffman, 2005). Maladaptive regulation of emotion could contribute to the development of anxiety and mood disorders and when people suppress their excitement out of habit, they experience more negative emotions, positive emotions less, social problems and a lower quality of life that makes person vulnerable against anxiety and mood disorders (Gross and John, 2003). Thus interfering emotion regulation with the control negative emotions and physical reactions and also by increasing internal control over their emotional and physical responses when facing with a stressful social situations (Hoffman, 2005) improve the symptoms of social phobia is the students. The second hypothesis of this study was: Meta-cognition strategies affect the reduction of student's social phobia. ANOVA was used to test the hypothesis. The test results indicated that the first hypothesis is confirmed $(\mathrm{P}<0.001)$, in other words, the results showed that meta-cognitive intervention has significantly reduced the symptoms of social phobia and physiological symptoms, fear and avoidance of social phobia in the study group.

The results of research conducted in this area show that Meta-cognitive beliefs in patients with anxiety disorders especially in generalized anxiety, panic, obsessivecompulsive and post-traumatic stress is more disturbed. Meta-cognition forms what we consider and enter the domain of our consciousness, evaluations and the effect of different strategies that we use to adjust our thoughts and feelings (Lobban et al, 2002) and amount of meta-cognitive and meta worries negative beliefs had a significant positive correlation with social phobia (Vaziri and Mousavi Nick, 2008). The results show anxiety is one of the basic components of anxiety disorders such as generalized anxiety disorder and social phobia which is particularly associated with negative and positive meta-cognitive beliefs (Hadyson and Ellis, 2010; quoted Bahadori et al., 2011). And meta-cognitive aspects have significant relationship with social anxiety (Espada, Jorju and Wells, 2010). Espada et al, (2012) consider metacognitive dimensions such as positive meta-cognitive beliefs about the concern and low cognitive trust as the causes of the stress and anxiety. Results of studies show meta-cognitive therapy has improved the status of generalized anxiety disorder and the improvement rate after implementing this type of treatment was 87.5 percent (Wells and King, 2006). And it has a significant effect in reducing the symptoms of social phobia (Bahadori et al., 2011). On the other hand, results show meta-cognitive intervention improves automatic social cognitive tendencies, accuracy and justice, as well as reflect on how the cognitive processes of life (Joshua and David, 2014). Meta-cognitive strategies with control the activities of cognitive systems, increase or reduce thinking strategies and contribute to an increase in regulatory processes. In anxiety disorders that mental events are often interpreted as a sign of mental collapse (For example, social anxiety disorder), among these meta-cognitive strategies with control thoughts and memory phenomena that have been assessed as negative and deal with catastrophic assessments 
and invasion of negative thoughts (Nelson et al., 1998) and reduces the symptoms of social anxiety.

The third hypothesis of this study was: Effectiveness of emotion regulation strategies with meta-cognitive are different in reducing student's social phobia. Tukey test was used to test the hypothesis. Tukey test showed there are significant differences between the control and two experimental groups which indicates a significant effect of these two methods in reducing the symptoms of social phobia in the experimental groups compared to the control group. Also results of Tukey test for comparison of two methods emotion regulation and meta-cognition in reducing the symptoms of social phobia showed that emotion regulation intervention in variables such as social phobia, fear, avoidance and psychological symptoms have almost the same effect than meta-cognitive intervention in two groups and Toki test results was not significant for it $(\mathrm{P}$ $>0.001$ ), in other words, both methods reduce social anxiety scores of the two groups and effectiveness of the two methods was not much difference. And statistically there was a not significant difference between the two groups. The results of research conducted in this area show the purpose of metacognitive therapy is increasing the supervision of person on the data processing and the dynamics of his thought, especially in stressful situations (Wells, 2000). Due to the nature of social anxiety disorder which is accompanied with emotional cognitive engagement of individuals, treatments planning that first stop emotional processing and then pay to meta-cognitive challenge can be very helpful. Meta-cognitive therapy methods actually pursue these objectives (Wells, 2003). People with social anxiety disorder, often before, during and even after the confrontation with a scary situation engage in mental rumination regarding their failure and how being seen from others and these rumination keeps their anxiety. While avoiding the feared situation in anxiety disorders, leads to the discovery of cases not dangerous. Also, more subtle forms of protective behaviors in people with social anxiety disorder such as to avoid exposing themselves to the opposite sidecar use to others react negatively and this leads to a sequence of negative social reaction (Wells et al., 2001). Both methods of emotion regulation and meta-cognition due to the nature of the therapeutic can help people with social phobia and with reducing of intellectual rumination, stop stream of defective thoughts and negative emotions reduce the symptoms of social phobia in the students. Therefore, given the nature of both methods and homogeneous groups the results of both methods was not much difference. The study also like other works was associated with restrictions including: Inability to control the variables associated with the individual, not using interviews and Rosenthal effect. In line with the limits, it is recommended variables associated with individual control in future studies and clinical interview should be used for the diagnosis of social phobia.

\section{Limitation}

Study on a larger statistical pool is advised.

\section{Authors' Contributions}

$\mathrm{MH}$ and MBM conceived and designed the evaluation. MBM performed the statistical analysis. SS and AE drafted the manuscript. All authors read and approved the manuscript.

\section{Acknowledgement}

We are very grateful to all participants for their cooperation in this study.

\section{References}

[1] American Psychiatric Association. (2000). Diagnostic and statistical manual of mental disorders (DSM-IVTR). 4th ed. Washington. DC: American Psychiatric Association, (12), 676-85.

[2] Bahadori, MH., Jahan bakhsh, M., Jamshidi, A and Askari, K. (2011) The efficacy of metacognitive treatment on anxiety symptoms in patients with social anxiety disorder, Journal of Knowledge and Research in Applied Psychology, Vol 12, No. 4, Pp. 12-21.

[3] Barlow, D. H. (2002). Anxiety and its disorders: the nature and treatment of anxiety and panic, second edition. New York NY: The Guilford press.

[4] Delavare, A. (2006). Foundations of Research in the Social Sciences, Tehran: the publication of growth.

[5] Fathi-Ashtiani, A. (2013). Psychological tests, Tehran: The Besat Publication.

[6] Gross, J. J \& John, O. P. (2003). Individual Differences in Two Emotion Regulation Processes: Implications for Affect, Relationships, and Well-Being, Journal of Personality and Social Psychology, 85, 348-362.

[7] Gross, J. J. (2002). Emotion regulation: Affective, Cognitive, and Social Consequences. Psychopathology and Behavioral Assessment, 26, 41-54.

[8] Hofmann, S. (2005). Perception of control over anxiety mediates the relation between catastrophic thinking and social anxiety in social phobia. Journal of Behavior Research and Therapy, 43, 885-895.

[9] Hofmann, S. G \& Barlow, D. H. (2002). Social phobia (social anxiety disorder). In D. H. Barlow (Ed.). Anxiety and its disorders: The nature and treatment of anxiety and panic (2nd. ed., pp. 454-477). New York: The Guilford Press.

[10] João-M. F\&David-L, R. (2014). Social Cognition and Interaction Training: The Role of Meta-cognition, Journal of Social Cognition and Meta-cognition in Schizophrenia, 162151.

[11] Jocobs, M., Snow, J., Geraci, M., Vythilingam, M., Blair, R. J. $\mathrm{R} \&$ et al. (2008). Association between level of emotional intelligence and severity of anxiety in generalized social phobia. Journal of Anxiety Disorders, 22, 1487-1495.

[12] Kadivar, P. (2004). Educational Psychology, Eighth Edition, Tehran: The Samt Publication. 
[13] Kashdan, T. B., Barrios, V., Forsyth, J \& Steger, M. F. (2006). Experiential avoidance as a generalized psychological vulnerability: comparisons with coping and emotion regulation strategies. Journal of Behavior Research and therapy, 44, 1301-1320.

[14] Lobban, F., Haddock, E., Einderman, P \& Wells, A. (2002). The role meta-cognitive beliefs auditory hallucination. Journal of Personality and Individual Differences, 32 (6), 1351-1363.

[15] Mashhadi, A., Ghasempour, A., Akbari, E., IL Beygi, R. and Hassanzadeh, Sh. (2013) the role of anxiety sensitivity and emotional regulation in predicting students' social anxiety disorder, Journal of knowledge and Research in Applied Psychology, Vol. 14, Pp. 89-99.

[16] McDermott, M. J., Tull, M. T., Gratz, K. L., Daughters, S. B \& Lejuez, C. W. (2009). The role of anxiety sensitivity and difficulties in emotion regulation inposttraumatic stress disorder among crack/ cocaine dependent patients in residential substance abusetreatment. Journal of anxiety disorders, 23, 591-599.

[17] Momeni, M., Rezaei, F. and Gorji, Y. (2013), Effectiveness of meta-cognitive counseling on anxiety level of female students, Journal of Applied Psychology, Vol 14, No. 4, Pp. 101-107.

[18] Moses, L. J \& Baird, J. A. (2002). Meta-cognition. In: Wilson RA, Keil FC (Editors). The MIT encyclopedia of the cognitive sciences. 2nded. Cambridge: MIT Press; 187-205.

[19] Narimani, M., Ariapooran, S., Abolqasemi, A. and Ahadi, B. (2011). Effectiveness of training mindfulness and emotion regulation on physical welfare and chemical veterans psychological, Research Journal, Vol. 15, No. 5, Pp. 61-72.

[20] Nelson, T. O., Kruglanski, A. W \& Jost, T. J. (1998). Knowing thyself and others: Progress in metacognitive social psychology. In V. Y. Yzerbyt G. Lories \& B. Dardenne (Eds.), Meta-cognition: Cognitive and social dimensions. London: Sage.

[21] Rapee, R. M \& Spence, S. H. (2004). The etiology of social phobia: Empirical evidence and aninitial model. Journal of Clin Psychol Rev, 24 (5), 737-67.

[22] Rodebaugh, T. L \& Heimberg, R. G. (2008). Chapter 9Emotion Regulation and the Anxiety Disorders: Adopting a Self-Regulation Perspective. Springer.

[23] Spada, M. M., Caselli, G., Manfredi, C., Rebecchi, D., Rovetto, F., Ruggiero, G. M, Nikcevic, A. V \& Sassaroli, S. (2012). parental overprotection andmeta-cognitions as predictors of worry and anxiety. Journal of Behavioral cognitive psychotherapy, 38 (5), 629-637.

[24] Spada, M. M., Georgiou, G\& Wells, A. (2010). Therelationship among meta-cognitions, intentional control and social anxiety. Journal of Cognitive behavioral therapy, 39 (1), 64-71.

[25] Tull, M. T., Stipelman, B. A., Salters-Pedneault, K \&Gratz, K. 1. (2009). An examination of recent non-clinicalpanic attacks, panic disorder, anxiety sensitivity, and emotion regulation difficulties in the prediction of generalized anxiety disorder in an analogue sample. Journal of anxiety disorders, 23, 275282.

[26] Vaziri, SH. And Mousavi Nick, M. (2008). Relationship between negative meta-cognitive beliefs and meta worries with phobias, Journal of Thought and Behavior, Vol. 3, No. 10, Pp. 59-61.

[27] Wells, A. (2009). Meta-cognitive therapy for anxiety and depression. New York: Guilford: 1-22.

[28] Wells, A \& King P. (2006). Meta-cognitive therapy for generalize anxiety disorder: an open trial. Journal of Behavior Therapy and Experimental Psychiatry, 37 (3), 206-612.

[29] Wells, A. (2003). Anxiety disorders, meta-cognition and change. In: Leahy RL. (editor). Roadblocks in-cognitivebehavioral therapy: Transforming challenges into opportunities for change. New York: Guilford: 69-88.

[30] Wells, A \& Matthews, G. (1994). Attention and emotion, A clinical perspective. Hove: Erlbaum. 